\title{
Epidemiology and Etiology of In-Hospital Cardiac Arrest at The American University of Beirut Medical Center (AUBMC)
}

\author{
Ahmed Eltarras $^{1}$, Youssef Jalloul ${ }^{1}$, Ola Assaad ${ }^{1}$, Yara Yammine ${ }^{2}$, Nina Khatib ${ }^{2}$, Abdallah \\ Rebeiz $^{1}$, Mazen El Sayed ${ }^{1}$, and Marwan Refaat ${ }^{1}$ \\ ${ }^{1}$ American University of Beirut Medical Center \\ ${ }^{2}$ American University of Beirut
}

July 13, 2020

\begin{abstract}
Introduction: In-hospital cardiac arrest(IHCA) constitutes a significant cause of morbidity and mortality. we devised this study to shed some light on it to better inform both hospitals and policymakers. Methods: We analyzed retrospective data from 680 IHCAs at the American University of Beirut Medical Center between July 1st, 2016, and May 2nd, 2019. Sociodemographic variables included age, sex, and comorbidities in the Charlson Comorbidity Index(CCI). IHCA variables were the day of the week, time from activation to arrival, event location, initial cardiac rhythm, the total number of IHCA events, and the months and years of the IHCAs. We considered the return of spontaneous circulation(ROSC) and survival to discharge(StD) to be our outcomes of interest. Results: The incidence of IHCA was 6.58 per 1000 hospital admissions. Non-shockable rhythms were $90.7 \%$ of IHCAs. Most IHCAs occurred in the Closed care units $(87.9 \%)$ and on weekdays $(76.5 \%)$. ROSC followed $56 \%$ of the IHCAs. Only $5.4 \%$ achieved StD. Survival outcomes were not significantly different between the time of the day and were higher in cases with a shockable rhythm. ROSC wasn't significantly different between weekdays and weekends. however, StD was higher on weekdays. A high CCI was associated with decreased StD. Conclusion: The incidence of IHCA was high, and its outcomes were lower compared to other developed countries. Survival outcomes were better for patients who had a shockable rhythm and were similar between the time of the day. These findings may help inform hospitals and policymakers about the magnitude and quality of IHCA care in Lebanon
\end{abstract}

\section{Hosted file}

Final IHCA Manuscript.pdf available at https://authorea.com/users/342416/articles/469225epidemiology-and-etiology-of-in-hospital-cardiac-arrest-at-the-american-university-ofbeirut-medical-center-aubmc 